\title{
Module Development for the Phase-2 ATLAS ITk Pixel Upgrade
}

\section{Dai Kobayashi on behalf of ATLAS collaboration}

\author{
Kyushu University, \\ Fukuoka City, Japan \\ E-mail: dai.kobayashi@cern.ch
}

\begin{abstract}
Aвstract: For the high luminosity upgrade of the Large Hadron Collider (HL-LHC), the instantaneous luminosity is expected to reach unprecedented values, resulting in about 200 proton-proton interactions in a typical bunch crossing. To cope with the resultant increase in occupancy, bandwidth and radiation damage, the ATLAS Inner Detector will be replaced by an all-silicon system, the Inner Tracker (ITk). The innermost part of ITk will consist of a state-of-the-art pixel detector, with an active area of about $14 \mathrm{~m}^{2}$, which will provide tracking capability up to $|\eta|=4$. Detector requirements in terms of radiation hardness and occupancy, as well as thermal performance depend strongly on the distance from the interaction region. Therefore, the innermost layer will feature 3D silicon sensors, due to their inherent radiation hardness and low power consumption, while the remaining layers will employ planar silicon sensors with thickness ranging from $100 \mu \mathrm{m}$ to $150 \mu \mathrm{m}$. All hybrid detector modules will be read out by novel ASICs, implemented in $65 \mathrm{~nm}$ CMOS technology and thinned to $150 \mu \mathrm{m}$, which will be connected to the silicon sensors using bump bonding. With the recent arrival of the first readout chip prototype, the RD53A chip, prototype modules are being built to study sensor and chip properties, thermal performance, as well as bump bonding yield in lab measurements and beam test campaigns. Irradiation studies are ongoing. The report will present latest results from the module characterization measurements both before and after irradiation.
\end{abstract}




\section{Contents}

1 ITk Pixel for HL-LHC 1

2 Radiation Tolerant Sensor Development 2

3 Rapid-Readout ASIC Development: RD53 Project 2

4 Module Assembly and Relating Components Study 3

5 Conclusion 4

\section{ITk Pixel for HL-LHC}

The High Luminosity LHC (HL-LHC) project aims at achieving better sensitivity to Beyond the Standard Model signatures and better precision on the Standard Model parameters than the present LHC machine by a considerable increase of the instantaneous luminosity. The target integrated luminosity is $4000 \mathrm{fb}^{-1}$. On the other hand, this upgrade requires higher radiation tolerance for detectors than ever. Therefore, the inner tracker of ATLAS detector will be replaced by an all-silicon system, the Inner Tracker (ITk). ITk, the pixel detector, is required to have the highest radiation tolerance among all sub-detectors. An ITk Pixel module consists of flip-chipped sensor with FrontEnd (FE) chips and Flexible Printed Circuit (FPC). Each pixel on the sensors is connected to the FE channel by bump bonding and the FPC is connected to the FE chips ( 2 or 4 per module) by $25 \mu \mathrm{m}$ diameter Aluminum wires for signal transmission. All wire-bonding will be protected by encapsulation using some soft compound. All wires are planed to be protected by encapsulation using soft compound. Fig. 1 shows a schematic drawing of the module.

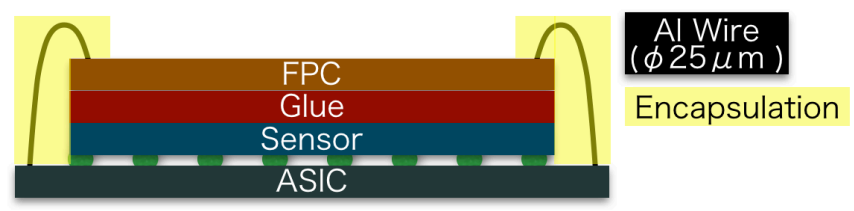

Figure 1. Schematic drawing of the module (side view).

One of the major issue at the HL-LHC is the radiation damage of sensors, FE chips, and materials. The total fluence is expected to be up to $10^{16} \mathrm{n}_{\mathrm{eq}} / \mathrm{cm}^{2}$. Performance and robustness of all components of the detector modules have to be established, including glues, FPC, and other mechanical components. Other critical requirements must be fulfilled in terms of readout speed and track multiplicity. In particular, the FE chips must sustain a $\mathrm{GHz}$ readout rate and the pixel size of the sensor and of the FE chips must be very small. Studies and developments about each part are proceeding in parallel, and are described below. 


\section{Radiation Tolerant Sensor Development}

Two types of pixel sensors are developed to balance the required radiation tolerance, varying across the detector volume, with the production costs. The planar-type sensor (sketched in Fig. 2(a)) comes from a rather standard production process. The $n^{+} i n-p$ structure has been chosen since it was shown to be more robust against type conversion than the $\mathrm{n}^{+}$-in- $n$ type.

The second type is the 3D-sensor, where n-type and p-type pillars runs vertically across the sensor (see Fig. 2(b)). The radiation tolerance of this structure is better than that of planar sensors because of the shorter collection distances. However, the production process is technically much more complex and expensive than in the case of planar sensors. This is the reason why the 3D sensor is not suitable for mass production with large number of modules. For the ITk upgrade, the 3D sensor has been developed for use in the innermost layer, while the planar one will be used in the other parts. The thickness of both types is small compared to previous generation pixel detectors, being $150 \mu \mathrm{m}$ or $100 \mu \mathrm{m}$. About the pixel size, there are two options: $50 \times 50 \mu \mathrm{m}^{2}$ or $25 \times 100 \mu \mathrm{m}^{2}$. The final choice will be made based on studies of tracking performance.

The performance of these sensors has been evaluated for different values of the thickness and pixel size. Planar and 3D sensors showed efficiency above 95\% even after irradiation. The results are shown as Fig. 3, summarized as the Technical Design Report [1].

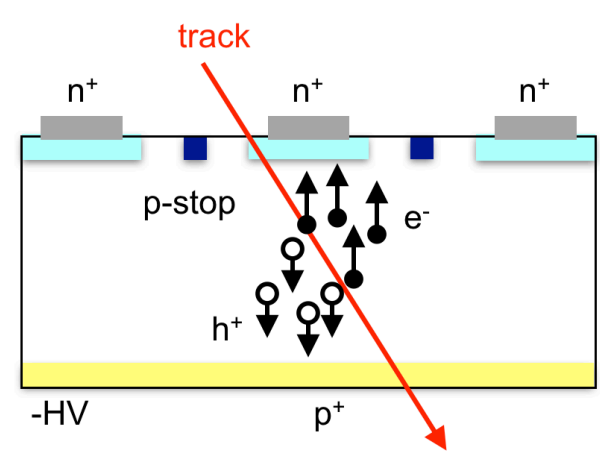

(a)

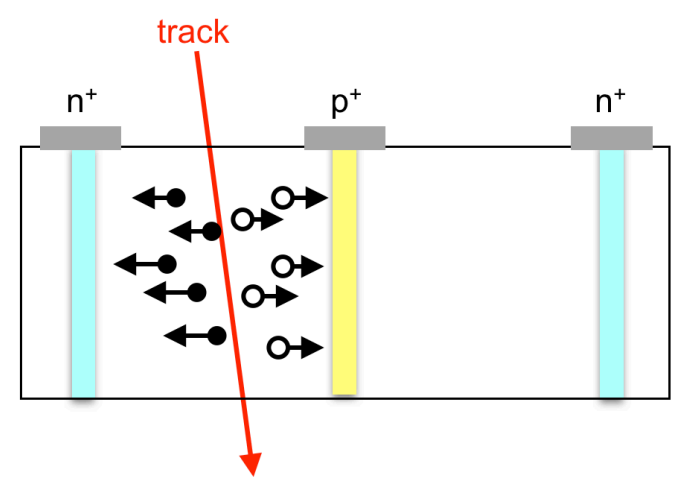

(b)

Figure 2. Descriprion of planar type sensor (a) and 3D type sensor (b).

\section{Rapid-Readout ASIC Development: RD53 Project}

FE chips for the high rate collisions at the HL-LHC are required to achieve rapid readout with few GHz. The RD53 collaboration is developing a new type of FE chip for the ATLAS and CMS experiments at the HL-LHC [2] based on $65 \mathrm{~nm}$ CMOS technology.. The target performance is a low noise rate with low threshold $\left(<10^{-6}\right.$ at $\left.600 \mathrm{e}^{-}\right)$with a data transfer rate of $1.28 \mathrm{GHz}$. The latest chip, called RD53A, has been designed in such a way to allow the comparison of three different implementations of the analog frontend as shown in Fig. 4. The sharp process line is expected to suppress the high rate of Single Event Upset (SEU), which is caused by a bit flip by injected particles into FE-chips. The evaluation of the efficiency and general features is ongoing by testbeam and irradiation. 


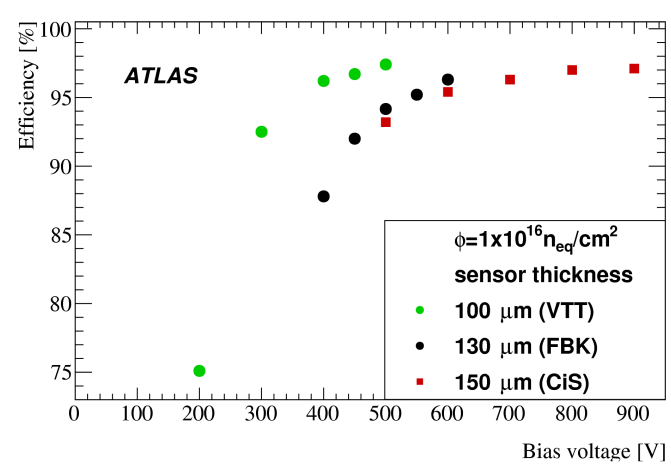

(a)

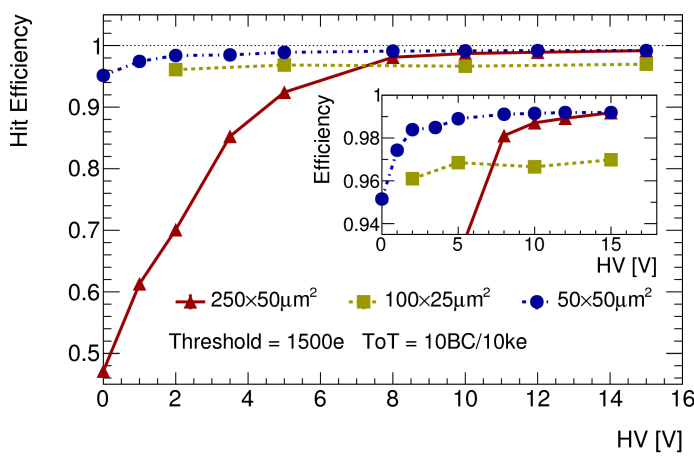

(b)

Figure 3. Efficiency as a function of the bias voltage for different sensor thicknesses (a) and different pixel sizes (b) [1].

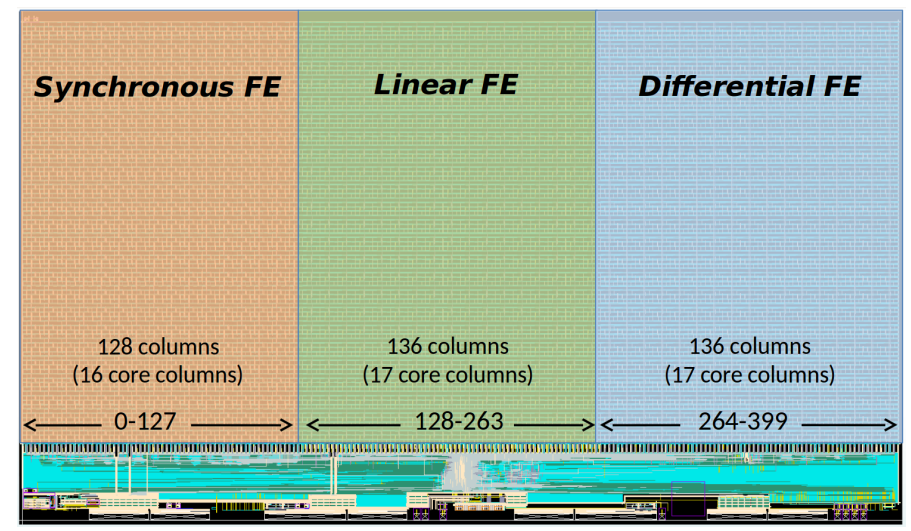

Figure 4. Three different layouts of the analog frontend are implemented in three different regions of the RD56A chip, in order to allow a direct comparison of the performance and guide the final choice[1].

\section{Module Assembly and Components Study}

In this phase, not only the development and performance study of new sensors and FE chips, but also deep scrutiny of the production techniques of the module are necessary. One of the most challenging task is the Bump-Bonding (BB) technique to connect the sensor and FE chips with 50 or $25 \mu \mathrm{m}$ pitch. This has been achieved for several modules so far, however, technical development is still ongoing to ensure higher yield in the mass production phase.

Module assembly procedure is also important for mass production. This procedure consists of the following steps: glueing of a flip-chipped sensor and a FPC, wire bonding between the FE chips and the attached FPC, and encapsulation of the wires. The sequence has already been performed at several assembly sites, and the optimization for higher yield and production rate is ongoing. Fig. 5 shows one of the mudules assembled with the techniques developed so far. In addition, the Quality Control (QC) is another important task to be accomplished at the assembly sites to ensure the module performance. The figures of merits to be established and recorded by the QC and the procedures to establish them are under discussion these days. 


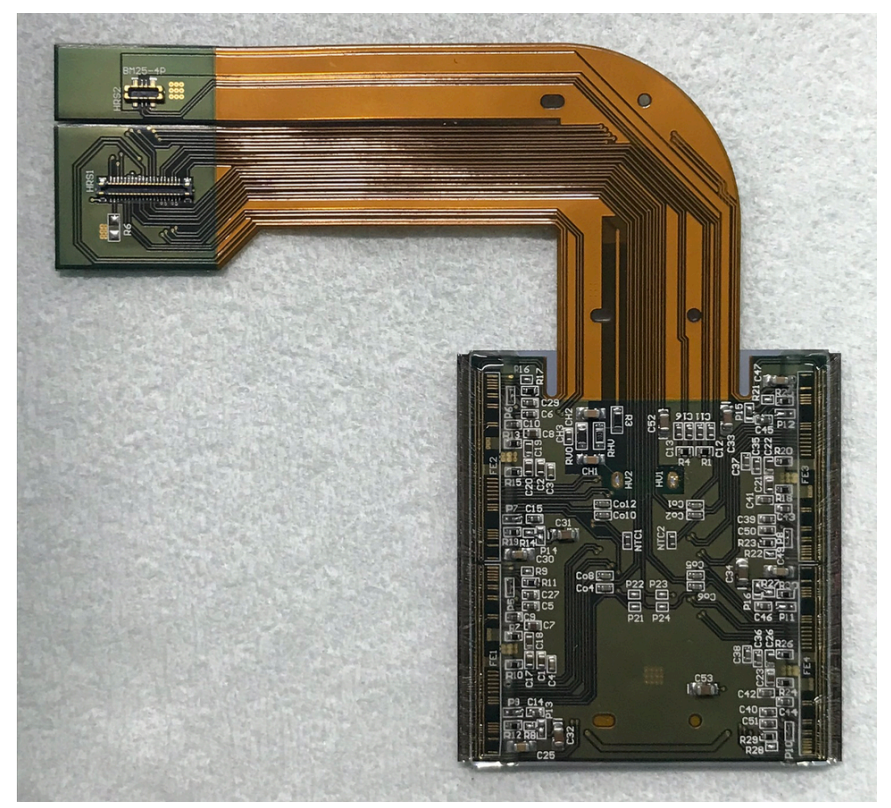

Figure 5. One of the assembled module composed of a sensor flip-chipped to four FE chips and a FPC with wire bonding and encapsulation.

The tolerance to high radiation dose of module components has to be evaluated. The radiation tolerance has been evaluated by irradiation test using gamma ray source and proton beam. The thermal cycling tolerance has been also tested for several components. As a result, it turns out that some compounds and glues can be used in the HL-LHC environment.

\section{Conclusion}

The HL-LHC is an important opportunity for high energy physics research, but the detector upgrade is necessary to cope with the radiation environment over the long data taking time. The ATLAS collaboration has developed new types of sensors and FE chips, for the new pixel detector, and their assembly procedures. Two types of $\mathrm{n}^{+}$-in-p sensors, planar and 3D sensor, have been developed for higher radiation tolerance and their design performance has been verified on beam test data. New FE chip development is ongoing for rapid readout with $1.28 \mathrm{GHz}$ and high radiation tolerance at the ATLAS and CMS experiments. In view of mass production of the modules, the study about module assembly process has been developed and optimization for higher yield is ongoing. The radiation tolerance of module components have been certified for use at $10^{16} \mathrm{n}_{\mathrm{eq}} / \mathrm{cm}^{2}$.

\section{References}

[1] ATLAS Collaboration, Technical Design Report for the ATLAS Inner Tracker Pixel Detector, CERN-LHCC-2017-021.

[2] RD-53 Collaboration, http://rd53.web.cern.ch/rd53/. 